Tropical Journal of Pharmaceutical Research April 2018; 17 (4): 715-721

ISSN: $1596-5996$ (print); 1596-9827 (electronic)

(C) Pharmacotherapy Group, Faculty of Pharmacy, University of Benin, Benin City, 300001 Nigeria.

\title{
Inappropriateness of medication use and associated health risks: A cross-sectional study from Pakistan
}

\author{
Syed Zia Husnain', Nadeem Irfan Bukhari', Khalid Hussain', Zaheer-ud-Din \\ Baber $^{2,3}$, Zikria Saleem ${ }^{1,4}$ \\ ${ }^{1}$ University College of Pharmacy, University of the Punjab, Allama lqbal Campus, Lahore 54000, Pakistan, ${ }^{2}$ Department of \\ Pharmacy, University of Huddersfield, HD1 3DH, Huddersfield, United Kingdom, ${ }^{3}$ School of Pharmacy, University of Auckland, \\ Private Mail Bag 92019, Auckland, New Zealand, ${ }^{4}$ School of Pharmaceutical Sciences, Universiti Sains Malaysia, Penang, \\ Malaysia
}

*For correspondence: Email: ziafid43@hotmail.com

Sent for review: 19 December 2017

Revised accepted: 25 March 2018

\begin{abstract}
Purpose: To evaluate the patterns of medication use and medication appropriateness among people in different regions of Pakistan.

Methods: A cross-sectional study involving 410 individuals in four provinces of Pakistan (Punjab, Sindh, Baluchistan and Khyber Pakhtunkhwa) as well as the capital city, Islamabad, via a convenient sampling method. The questionnaire used comprised three major sections: (a) medication use patterns of the population, (b) medication storage and wastage practices, and (c) development of secondary disease after medication use. The questionnaires were completed under the supervision of pharmacists trained for the purpose.

Results: The majority of the study population preferred bottle caps for taking oral liquid medicines (83.9 $\%)$, and the proportion using bottle caps was highest in Punjab (55.2\%) followed by Sindh (21.8\%). A significant proportion of the population consider the use of very hot water as a reconstitution medium for suspensions (36.3\%). In many cases, suspensions were constituted by mere estimation of the vehicle used (56.1\% from Punjab, 18.9 \% from Sindh, $16.1 \%$ from KPK, $7.7 \%$ from Baluchistan, and $1.1 \%$ from Islamabad). Significant differences were observed in the handling of leftover drugs among respondent populations, as well as the overall diverse modes of disposal recorded $(43.9 \%)$. Doctors $(46.6 \%)$ and relatives were the main sources of instructions on medicine usage (37.6\%). A disinclination to purchase a full course of medicine was observed. Knowledge of medicine storage and measuring tools for oral liquids showed striking peculiarities among the various study populations.

Conclusion: Inappropriate medication use among the general population of Pakistan requires educational interventions and awareness programs to promote rational drug use in the society.
\end{abstract}

Keywords: Inappropriate, medication, population, healthcare, use

This is an Open Access article that uses a funding model which does not charge readers or their institutions for access and distributed under the terms of the Creative Commons Attribution License (http://creativecommons.org/licenses/by/4.0) and the Budapest Open Access Initiative (http://www.budapestopenaccessinitiative.org/read), which permit unrestricted use, distribution, and reproduction in any medium, provided the original work is properly credited.

Tropical Journal of Pharmaceutical Research is indexed by Science Citation Index (SciSearch), Scopus, International Pharmaceutical Abstract, Chemical Abstracts, Embase, Index Copernicus, EBSCO, African Index Medicus, JournalSeek, Journal Citation Reports/Science Edition, Directory of Open Access Journals (DOAJ), African Journal Online, Bioline International, Open-J-Gate and Pharmacy Abstracts

\section{INTRODUCTION}

Notwithstanding the importance of essential medicines at the healthcare level, rational use of drugs worldwide is vital, as more than $50 \%$ of medicines are inappropriately prescribed or dispensed and $50 \%$ of consumers use medicines inappropriately [1]. Self-medication, non-compliance, not sticking to prescribers' guidelines, unsafe and overuse of medicines are 
some of the determinants of inappropriate medication use by the consumers [2].

Medication errors committed by the patient/caregiver have been extensively reported by several studies [3,4]. A study reported $41 \%$ medication errors based on dosing where counseling provided by the healthcare professionals and provision of dosing tools were helpful in reducing the dosing errors [5]. One of the major factors leading to incorrect use of medicines is low literacy of patients/patients' attendants [6]. Other factors include polypharmacy, co-morbidities, and age-related physiological changes that hamper patients' understanding of the use of the drug [7]. Among other major determinants of irrational drug use, self-prescribing of the prescription drugs such as antibiotics cannot be neglected and should be discouraged, to reduce the health and cost burden on the patient, and optimize the drug use in the community [8]. A study from Oman showed that $43 \%$ of the population practiced selfmedication while $70 \%$ stopped using medicine after the disappearance of symptoms [9].

Patient information, improved drug packaging and labeling, updated knowledge among pharmacy staff and appropriate communication between the patient and healthcare practitioners are factors that can effectively control medication mismanagement and out-of-pocket expenditures for the patient [10]. To the best of our knowledge, no study regarding the appropriateness of medication use has been previously performed in Pakistan.

Therefore, the current study is aimed at evaluating medication usage patterns and medication appropriateness among individuals living in different regions of Pakistan.

\section{METHODS}

\section{Study design}

A cross-sectional questionnaire-based study was carried out from March 2015 to January 2016 to investigate the pattern of drug use among the general population of Pakistan.

\section{Ethical approval}

Ethical approval for the study was obtained from the Ethical Committee of Clinical Research, Punjab University College of Pharmacy, University of the Punjab, Lahore, Pakistan, with ref no. D/HEC1000/UCP01920. Informed verbal and written consent was obtained from the study participants before the initiation of the study. The ethical principles of the Declaration of Helsinki were followed for the study [11].

\section{Study participants}

A sample size of $\mathrm{n}=385$ was calculated using online sample calculator Raosoft with $95 \%$ confidence interval and $5 \%$ margin of error keeping in view the total population of Pakistan [12]. An additional $6.0 \%$ sample size was added to compensate for errors/partially filled/unfilled questionnaires. Therefore, a final sample size (n) of 410 was taken for the study. The study population was selected via a convenient sampling approach from healthcare facilities and subsequently drug utilization practices were evaluated at the homes of the study population through a questionnaire.

\section{Inclusion and exclusion criteria}

Conscious, mentally stable individuals were included in the study. Those having Pakistani nationality were included. Immigrants and mentally unstable people were excluded.

\section{Data collection tool and administration}

A comprehensive questionnaire was designed after extensive literature review by the authors. The content validation and face validation of the questionnaire was performed by two experts, NIB \& $\mathrm{KH}$ from the University of the Punjab, Lahore, Pakistan, who are experienced in conducting quantitative studies especially in the areas of medication use, pharmacy practice and pharmaceutical policy. The questionnaire was upgraded and improved according to the propositions and remarks received. The questionnaire comprised three major sections:

(a) Medication use patterns of the population, (b) Medication storage and wastage practices, and (c) Development of secondary disease after medication use. The study was conducted in four provinces (Punjab, Sindh, Baluchistan and Khyber Pakhtunkhwa) of Pakistan and Islamabad, the capital city. Questionnaires were completed under the supervision of trained pharmacists from the University of the Punjab, Lahore, Pakistan. The study participants were told about the objectives of the study at the beginning and were counseled by the pharmacists about appropriate use of the drugs at the end.

\section{Statistical analysis}

Data were analyzed using Statistical Program for Social Sciences (SPSS). 


\section{RESULTS}

Table 1 depicts the knowledge and perception of the population and the medication use patterns. On the basis of the population responding to the respective query options; $55.2 \%$ of the population living in the Punjab region were of the view that medicine should be taken with a bottle cap. The population of Islamabad agreed with the use of spoon as a mode of taking medicine, yielding significant results. $60.7 \%$ of responding individuals from Punjab used boiled cooled water as a reconstitution liquid whereas $53 \%$ used very hot water to reconstitute liquid for suspensions. Users of milk as a reconstitution medium mainly lived in the Punjab (47.2\%) and Sindh $(22.6 \%)$ regions. The overriding approach of the overall study population was to use very hot water for reconstitution (36.3\%). To the query option of using the line of a bottle to make reconstitution suspensions: more than half $(58.8$ $\%$ ) of the respondents were from Punjab, and responses from Sindh and Islamabad were better
(17.6 \% and $13.7 \%$ respectively) compared to KPK and Balochistan (5.9 \% and $3.9 \%$ respectively). Overall $(n=410)$, over two-thirds of the population followed the poor practice of making reconstitution suspensions by mere estimation (69.5\%). Surprisingly, $71.4 \%$ of respective answering participants from Punjab used leftover drugs for other indications and many of them $(50.5 \%)$ stored drugs for future use or gave them to others (60\%). Many individuals from Sindh wasted the leftover drugs (25.3 \%). Most study participants used medication on the advice of a doctor (46.6\%), though a marked number also considered the advice of relatives (37.6\%). The practices of drug disposal and medication usage are shown in Table 1.

The storage practices and knowledge of measuring volumes as a basic spoonful are shown in the Table 2. $64.4 \%$ of all the participants did not purchase the full course of medicine.

Table 1: Knowledge and medication use patterns

\begin{tabular}{|c|c|c|c|c|c|c|}
\hline Parameter & Punjab & Sindh & KPK & Balochistan & Islamabad & $\begin{array}{l}\text { Overall population } \\
\text { response }(n=410)\end{array}$ \\
\hline \multicolumn{7}{|l|}{ Mode of taking medicine } \\
\hline$\overline{\text { With bottle }}$ & $18(66.7)$ & $4(14.8)$ & $3(11.1)$ & $1(3.7)$ & $1(3.7)$ & $27(6.6)$ \\
\hline With bottle cap & $190(55.2)$ & $75(21.8)$ & $52(15.1)$ & $24(7)$ & $3(0.9)$ & $344(83.9)$ \\
\hline Spoon & $18(46.2)$ & $5(12.8)$ & $3(7.7)$ & $4(10.3)$ & $9(23.1)$ & $39(9.5)$ \\
\hline \multicolumn{7}{|l|}{ Reconstitution liquid } \\
\hline$\overline{\text { Plain water }}$ & $31(56.4)$ & $10(18.2)$ & $10(18.2)$ & $3(5.5)$ & $1(1.8)$ & $55(13.4)$ \\
\hline Boiled, cooled water & $74(60.7)$ & $20(16.4)$ & $9(7.4)$ & $8(6.6)$ & $11(9)$ & $122(29.8)$ \\
\hline Very hot water & $79(53)$ & $35(23.5)$ & $22(14.8)$ & $12(8.1)$ & $1(0.7)$ & $149(36.3)$ \\
\hline Mineral water & $6(54.5)$ & $2(18.2)$ & $2(18.2)$ & $1(9.1)$ & $0(0)$ & $11(2.7)$ \\
\hline Juice & $11(55)$ & $5(25)$ & $4(20)$ & $0(0)$ & $0(0)$ & $20(4.9)$ \\
\hline Milk & $25(47.2)$ & $12(22.6)$ & $11(20.8)$ & $5(9.4)$ & $0(0)$ & 53 (12.9) \\
\hline \multicolumn{7}{|l|}{ Making of suspension } \\
\hline Up to line of bottle & $30(58.8)$ & $9(17.6)$ & $3(5.9)$ & $2(3.9)$ & $7(13.7)$ & $51(12.4)$ \\
\hline By estimation & $160(56.1)$ & $54(18.9)$ & $46(16.1)$ & $22(7.7)$ & $3(1.1)$ & $122(29.8)$ \\
\hline Others & $36(48.6)$ & $21(28.4)$ & $9(12.2)$ & $5(6.8)$ & $3(4.1)$ & $149(36.6)$ \\
\hline \multicolumn{7}{|l|}{ Handling of leftover drugs } \\
\hline Waste & $50(60.2)$ & $21(25.3)$ & $7(8.4)$ & $3(3.6)$ & $2(2.4)$ & $83(20.2)$ \\
\hline Store for future use & $52(50.5)$ & $17(16.5)$ & $16(15.5)$ & $9(8.7)$ & $9(8.7)$ & $103(25.1)$ \\
\hline Given to others & $18(60)$ & $6(20)$ & $4(13.3)$ & $1(3.3)$ & $1(3.3)$ & $30(7.3)$ \\
\hline Use for other indication & $10(71.4)$ & $3(21.4)$ & $0(0)$ & $0(0)$ & $1(7.1)$ & $14(3.4)$ \\
\hline Others & 96 (53.3) & $37(20.6)$ & $31(17.2)$ & $16(8.9)$ & $0(0)$ & $180(43.9)$ \\
\hline \multicolumn{7}{|l|}{ Medicine used on } \\
\hline Doctor & $100(52.4)$ & $40(20.9)$ & $23(12)$ & $17(8.9)$ & $11(5.8)$ & $191(46.6)$ \\
\hline Pharmacist & $13(54.2)$ & $4(16.7)$ & $4(16.7)$ & $3(12.5)$ & $0(0)$ & $24(5.9)$ \\
\hline Relative & $93(60.4)$ & $30(19.5)$ & $23(14.9)$ & $6(3.9)$ & $2(1.3)$ & $154(37.6)$ \\
\hline Friend & $4(66.7)$ & $2(33.3)$ & $0(0)$ & $0(0)$ & $0(0)$ & $6(1.5)$ \\
\hline Others & $16(45.7)$ & $8(22.9)$ & $8(22.9)$ & $3(8.6)$ & $0(0)$ & $35(8.5)$ \\
\hline Disposal of remaining & & & & & & \\
\hline \multicolumn{7}{|l|}{ drug } \\
\hline Thrown away & $208(55.9)$ & $80(21.5)$ & $57(15.3)$ & $25(6.7)$ & $2(0.5)$ & $372(90.7)$ \\
\hline Thrown in street & $10(58.8)$ & $4(23.5)$ & $1(5.9)$ & $2(11.8)$ & $0(0)$ & $17(4.1)$ \\
\hline Dust bin & $7(100)$ & $0(0)$ & $0(0)$ & $0(0)$ & $0(0)$ & $7(1.7)$ \\
\hline Wasted in kitchen trash & $0(0)$ & $0(0)$ & $0(0)$ & $2(16.7)$ & $10(83.3)$ & $12(2.9)$ \\
\hline Others & $1(50)$ & $0(0)$ & $0(0)$ & $0(0)$ & $1(50)$ & $2(0.5)$ \\
\hline
\end{tabular}


Among them, the majority of the population was from Punjab (56.4\%) and Sindh (21.2\%). In contrast, the majority of people from Islamabad took the full course of prescribed medicines. 62.4 $\%$ of the population frequently stored medicines in the fridge, and $54.4 \%$ used the deep freezer to store medicines. In Islamabad, only $1.8 \%$ of the population stored medicines in the deep freezer, in contrast to Punjab (55.2\%) and Sindh $(20.2 \%)$. A large proportion $(44.4 \%)$ of the Punjab population did not know about the storage conditions of medicines followed by Sindh and KPK (22.2\% each) (Table 2).

Also, $36.1 \%$ of study participants considered 10 $\mathrm{ml}$ to be equal to one teaspoonful and $26.3 \%$ believed that $15 \mathrm{ml}$ was equal to one teaspoonful. The population of Islamabad had better consideration of measuring tools and only $2 \%$ and $1.9 \%$ had the wrong perception regarding the capacity of a teaspoon (believing it to be $10 \mathrm{ml}$ and $15 \mathrm{ml}$, respectively). A good number of people from Punjab had the idea of medicine storage temperature $(57.3 \%)$, though many did not know about it (55.2\%). People from Sindh were aware of storage practices while those from Balochistan and KPK did not have a basic idea of medication storage temperature as indicated in Table 2.

Table 3 demonstrates the development of secondary disease after inappropriate medication use. The results indicated that $55.7 \%$ of people in Punjab and a majority (16\%) of KPK population believed that inappropriate medication use leads to development of other ailments whereas the majority of study participants from Sindh (26.7 \%) and Balochistan region (10.0\%) did not know about it. People living in Islamabad were of the view that inappropriate medication use does not lead to development of secondary ailments as shown in Table 3.

Table 2: Knowledge about storage and measuring volumes

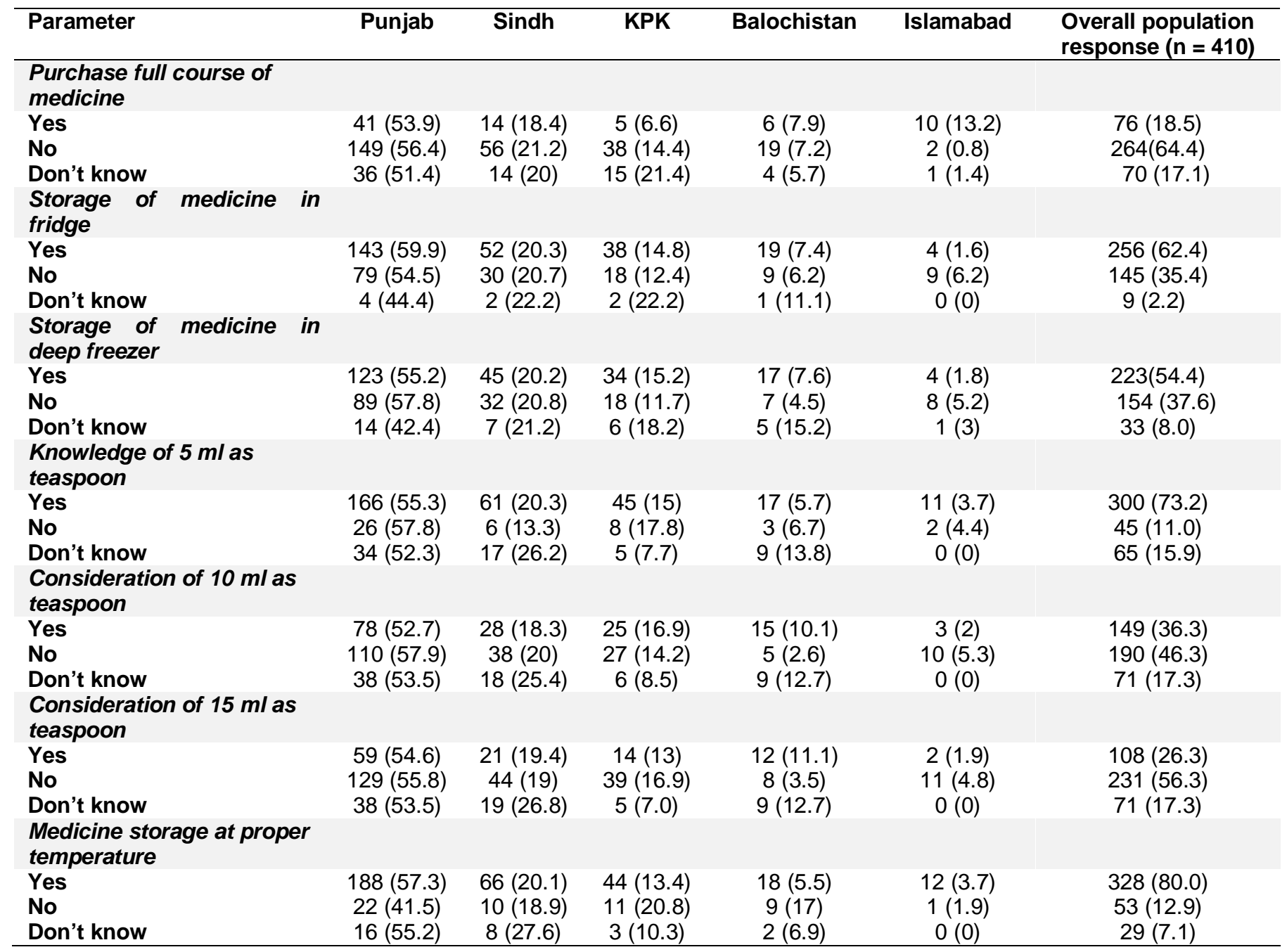


Table 3: Development of secondary disease due to inappropriate medication use

\begin{tabular}{|c|c|c|c|c|c|c|}
\hline Parameter & Punjab & Sindh & KPK & Balochistan & Islamabad & $\begin{array}{l}\text { Overall population } \\
\text { response }(n=410)\end{array}$ \\
\hline \multicolumn{7}{|c|}{$\begin{array}{l}\text { Secondary disease due to } \\
\text { inappropriate medication use }\end{array}$} \\
\hline Yes & $\begin{array}{c}73 \\
(55.7)\end{array}$ & $26(19.8)$ & $21(16.0)$ & $10(7.6)$ & $1(0.8)$ & $131(32)$ \\
\hline No & $\begin{array}{c}137 \\
(55.0)\end{array}$ & $50(20.1)$ & $34(13.7)$ & $16(6.4)$ & $12(4.8)$ & $249(60.7)$ \\
\hline Don't know & $\begin{array}{c}16 \\
(53.3)\end{array}$ & $8(26.7)$ & $3(10.0)$ & $3(10.0)$ & $0(0)$ & 30 (7.3) \\
\hline
\end{tabular}

ns $=$ non-significant

\section{DISCUSSION}

The results of the current study indicated that the majority of the population living in different regions of Pakistan has poor knowledge regarding appropriate medication use. More than half $(55.2 \%)$ of the people living in the Punjab region used liquid medicine with bottle caps. The knowledge of reconstitution of suspension was very poor among the study population indicating a strong need for patient education to minimize the hazards associated with inappropriate medication usage [13]. In Pakistan, the system of healthcare is not well developed in terms of the availability of qualified staff at drug stores and community pharmacies. Patients are dealt with by sales people who hardly know anything about the medicines. The sale of medicines must be supervised by a qualified pharmacist to optimize drug use [14].

This study also indicated that unused medicine storage practices were common among the study population and sharing of medicine with others was observed. A study from Sudan reported 55 $\%$ re-use of previously stored drugs, $46.9 \%$ selfmedication habits probably due to easy availability at home, $59.3 \%$ sharing of drugs among family members and $71.2 \%$ noncompliance with prescribed medicine among the patients [15]. Such inappropriate use of medicine can lead to hazardous health outcomes and frequent hospital admissions as a result of secondary ailments associated with the improper use of drugs [16]. Inappropriate medication use can also pose a major economic burden to the patient along with poor health outcomes [17].

Furthermore, the results of the current study indicate poor knowledge regarding storage of medicine, as many had the idea of storing medicine in refrigerators or deep freezers, irrespective of pharmaceutical storage temperature requirements. A study from Ireland showed that $90 \%$ of the population stored unused medicine at home and $75 \%$ disposed of the remaining medicine if adequately counseled, highlighting the role of healthcare practitioners in public education for rational use of drugs [18]. The current study also indicated poor pharmaceutical disposal practices among the study population. Standard medicine waste management policies along with collaborative efforts of pharmacists and other healthcare professionals to educate patients can fill the knowledge gaps of the public regarding medicine storage and disposal [19]. Some of the markers for inappropriate use of drugs among the individuals involve poor clinical practices, selfmedication habits and socio-economic factors [20].

Improvements in drug use by patients can be made through close monitoring of patient profiles, doing follow-ups, assessing the appropriateness of drugs for indication and considering other patient- and drug-related factors to prevent health hazards to patients [21]. Medication management on behalf of the user must be evaluated by healthcare practitioners in terms of expired medication, duplicate generics and non-compliance [22]. Trained staff along with qualified pharmacists at drug stores can help patients to better understand the usage and storage conditions of the medicine leading to patient safety and improvement of healthcare in the community [23]. Therefore, prescribers' education, patients' education, regulatory controls on easy access to medicine, information campaigns for the public and media awareness programs can help to reduce unnecessary and inappropriate use of drugs among the population [24].

\section{Limitations of the study}

The study had a few limitations. Firstly, the inability to validate the answers of study participants due to the subjective nature of the questionnaire was a major limitation, as respondents might have underestimated the value of correct medication usage. Secondly, the cross-sectional nature of the study is subjected to respondent recall bias. 


\section{CONCLUSION}

Persistent inappropriate use of medicine among the general population living in different regions of Pakistan indicates a strong need for public education on the appropriate use of drugs via educational interventions and awareness seminars. The findings of this study should aid government healthcare officials and policy makers to strategize and implement rational use of drugs in the country. It further highlights the role of healthcare professionals such as physicians, pharmacists and nurses in improving healthcare systems in the society.

\section{DECLARATIONS}

\section{Acknowledgement}

The authors are extremely thankful to the study population for their precious time and valuable participation in the study.

\section{Conflict of interest}

No conflict of interest is associated with this study. No funding or support was received for this study from any organization.

\section{Author contributions}

We declare that this work was done by the authors named in this article and all liabilities pertaining to claims relating to the content of this article will be borne by the authors. Designed the experiment: NIB, KH. Performed the study: SZH. Statistically analyzed the data: SZH, ZS. Wrote the paper: SZH, ZS. Critically revised the manuscript: ZB. All the authors revised the manuscript and made the necessary amendments for publication of final manuscript.

\section{REFERENCES}

1. Organization WH. Promoting rational use of medicines: core components. 2002.

2. Hardon A, Hodgkin C, Fresle D. Organization WH. How to investigate the use of medicines by consumers. 2004.

3. Shah K, Barker KA. Out-of-hospital medication errors: a6year analysis of the national poison data system. Pharmacoepidemiol Drug Saf, 2009. 18 (11): 10801085.

4. Crouch BI, Caravati EM, Moltz E. Tenfold therapeutic dosing errors in young children reported to US poison control centers. Am J Health Syst Pharm, 2009. 66 (14).

5. Yin HS, Dreyer BP, Moreira $H A$, van Schaick $L$, Rodriguez L, Boettger S, Mendelsohn AL. Liquid medication dosing errors in children: role of provider counseling strategies. Acad Pediatr, 2014. 14 (3): 262270.

6. Wolf MS, Davis TC, Tilson HH, Bass III PF, Parker RM. Misunderstanding of prescription drug warning labels among patients with low literacy. Am J Health Syst Pharm, 2006. 63 (11).

7. Fialová $D$, Onder $G$. Medication errors in elderly people: contributing factors and future perspectives. $\mathrm{Br} J \mathrm{Clin}$ Pharmacol, 2009. 67 (6): 641-645.

8. Saleem Z, Saeed H, Ahmad M, Yousaf M, Hassan HB, Javed A, Anees N, Maharjan S. Antibiotic SelfPrescribing Trends, Experiences and Attitudes in Upper Respiratory Tract Infection among Pharmacy and NonPharmacy Students: A Study from Lahore. PLoS One, 2016. 11 (2): e0149929.

9. Abdo-Rabbo A, Al-Ansari M, Gunn BC, Suleiman BJ. The use of medicines in Oman: public knowledge, attitudes and practices. Sultan Qaboos Univ Med J, 2009. 9 (2): 124.

10. Anderson P. Medication errors: Best Practices.

11. Association WM. World Medical Association Declaration of Helsinki. Ethical principles for medical research involving human subjects. Bull World Health Organ, 2001. 79 (4): 373.

12. Population Census / Pakistan Bureau of Statistics.

13. Ikeda $Y$. Importance of patient education on home medical care waste disposal in Japan. Waste management, 2014. 34 (7): 1330-1334.

14. Hussain A, Ibrahim MIM. Medication counselling and dispensing practices at community pharmacies: a comparative cross sectional study from Pakistan. Int $\mathrm{J}$ Clin Pharm, 2011. 33 (5): 859-867.

15. Yousif $M$. In-home drug storage and utilization habits: a Sudanese study. 2002.

16. Sehgal V, Bajwa SJS, Sehgal R, Bajaj A, Khaira U, Kresse V. Polypharmacy and potentially inappropriate medication use as the precipitating factor in readmissions to the hospital. Journal of family medicine and primary care, 2013. 2(2): p. 194.

17. Ladd AM, Panagopoulos G, Cohen J, Mar N, Graham R. Potential costs of inappropriate use of proton pump inhibitors. The American journal of the medical sciences, 2014. 347 (6): 446-451.

18. Vellinga A, Cormican S, Driscoll J, Furey M, O'Sullivan M, Cormican M. Public practice regarding disposal of unused medicines in Ireland. Sci Total Environ, 2014. 478: 98-102.

19. Bashaar M, Thawani V, Hassali MA, Saleem F. Disposal practices of unused and expired pharmaceuticals among general public in Kabul. BMC Public Health, 2017. 17 (1): 45.

20. Fialová D, Topinková E, Gambassi G, Finne-Soveri $H$, Jónsson PV, Carpenter I, Schroll M, Onder G, Sørbye LW, Wagner C. Potentially inappropriate medication use among elderly home care patients in Europe. JAMA, 2005. 293 (11): 1348-1358. 
21. Scott IA, Hilmer SN, Reeve E, Potter K, Le Couteur D, Rigby D, Gnjidic D, Del Mar CB, Roughead EE, Page A. Reducing inappropriate polypharmacy: the process of deprescribing. JAMA internal medicine, 2015. 175 (5): 827-834.

22. Sorensen L, Stokes J, Purdie D, Woodward M, Roberts MS. Medication management at home: medication risk factor prevalence and inter-relationships. J Clin Pharm Ther, 2006. 31 (5): 485-491.

23. Haynes KT, Oberne A, Cawthon C, Kripalani $S$. Pharmacists' recommendations to improve care transitions. Ann Pharmacother, 2012. 46 (9): 1152-1159. 24. Lowe M. Strategies to Reduce the Supply of Prescription Drugs. 2017. 